\title{
Niños jugando a los dados de Pedro Núñez de Villavicencio. Historia de una obra a través de su radiografía
}

\author{
Laura Alba y Maite Jover de Celis
}

Resumen: La radiografía de la obra Niños jugando a los dados de Pedro Núñez de Villavicencio ha aportado interesantes datos sobre su historia material. Además de constatar la existencia de un añadido histórico de mano de Luca Giordano en la parte superior, cuya técnica se distingue fácilmente de la de Villavicencio, se revelan varios arrepentimientos e incluso una reutilización del lienzo original. Por debajo de la escena de niños y en perpendicular al sentido de la misma, se descubre el retrato de cuerpo entero de un caballero con una gran Cruz de Malta en el pecho. Teniendo en cuenta la biografía del pintor, se propone a una hipótesis razonable sobre la personalidad del retratado así como el posible formato original de la pintura oculta.

Palabras clave: Pedro Núñez de Villavicencio, radiografía, Niños jugando a los dados, Museo del Prado, estudio técnico, Luca Giordano, Manuel Arias, Orden de Malta.

\begin{abstract}
The painting Niños jugando a los dados (Children playing dice), painted by Pedro Núñez de Villavicencio, has been x.-rayed and the resulting image shows very interesting results. The existence of a historical added strip by Luca Giordano in the upper area, where a different painting technique can be distinguished, has been confirmed and many pentimienti can be detected as well, even a canvas reused. Under the children scene, in a perpendicular direction, a gentleman full length portrait wearing a big Malta Cross can be seen. Bearing in mind the painter's biography, a reasonable hypothesis about the depicted gentleman is presented, as well as a possible original format interpretation.
\end{abstract}

Keywords: Pedro Núñez de Villavicencio, X-ray photography, Children playing dice, The Prado Museum, Technical study, Luca Giordano, Manuel Arias, Malta Cross.

Resumo: A radiografia da obra Niños jugando a los dados de Pedro Núñez de Vilavicencio aportou interessantes dados sobre a sua história material. Para além de constatar a existência de um aporte histórico da mão de Luca Giordano na parte superior, cuja têcnica distingue-se fácilmente da de Vilavicencio, revelam-se vários arrependimentos e inclusive uma reutilização do lenço original. Por debaixo da cena das crianças, e em perpendicular ao sentido da mesma, descobre-se o retrato do corpo inteiro de um cavaleiro com uma grande Cruz de Malta no peito. Tendo em conta a biografia do pintor, propõe-se uma hipótese razoável sobre a personalidade do retratado, assim como o possível formato original da pintura oculta.

Palavras-chave: Pedro Núñez de Vilavicencio, radiografia, Niños jugando a los dados, Museu do Prado, estudo têcnico, Luca Giordano, Manuel Arias, Ordem de Malta.

\section{El artista}

Pedro Núñez de Villavicencio (Sevilla h. 1635-Madrid, 1695) fue un destacado pintor entre los seguidores de Murillo, siendo precisamente su obra más conocida Niños jugando a los dados, del Museo del Prado (P-01235). A pesar de ser pocas las pinturas atribuidas con certeza a su mano, su vida es bien conocida en comparación con la de otros artistas debido a la proyección pública que ésta tuvo ${ }^{1}$, con una intensa actividad política.

La historia personal de Pedro Núñez de Villavicencio resulta decisiva a la hora de entender la evolución de su técnica artística. A diferencia de los artistas de su entorno, proviene de familia noble -su padre era un almirante sevillano- y su posición social le permitió una vida desahogada y 
sobre todo la posibilidad de viajar y conocer otros corrientes estéticas. Estas experiencias van enriqueciendo su técnica, incorporando nuevas influencias a su estilo inicial adquirido en el círculo de Murillo, con quien le unió una relación de amistad hasta el momento de la muerte del maestro en 1682.

A finales de 1661 es admitido como Caballero de Justicia en la Orden de Malta lo que da inicio a su carrera como alto funcionario. Durante su obligada estancia en la isla, toma contacto con el italiano Mattia Preti, de quien según Palomino, se convierte en discípulo y copia numerosas obras (Palomino, 1988: 198) ${ }^{2}$. Posiblemente a su vuelta de Malta o en alguna estancia breve en Sevilla pinta el celebrado retrato del arzobispo de esta diócesis, don Ambrosio Ignacio de Spínola, que da fe de su buen hacer en este género ${ }^{3}$. Las influencias del barroco italiano que recibe en su estancia maltesa se completarán con visitas a otras ciudades italianas, como Roma, a donde se desplaza en 1673. Posiblemente también viaja a Nápoles y Sicilia, siempre en misiones relacionadas con su carrera política, donde conocerá la pintura de Giordano.

De vuelta a España hacia a 1675, desde Sevilla se entrega a la administración de fincas propiedad de la orden de Malta, mientras continúa con su actividad artística que, a pesar de no ser su principal fuente de ingresos, nunca abandona. Según Palomino, continúa siendo gran amigo de Murillo e incluso es nombrado por éste como albacea testamentario a su muerte.

En 1689 es nombrado Comendador de Bodonal de la Sierra, en la provincia de Badajoz (González Ramos, 1999:234). Este hecho es relevante en cuanto a que aparece reflejado en la firma de Niños jugando a los dados y nos permite fijar una fecha aproximada para la realización de la obra ${ }^{5}$. Según Palomino, el pintor regaló al rey este cuadro, posiblemente en agradecimiento por el nombramiento, lo que explicaría una firma tan poco habitual, que no aparece en ninguna otra de sus obras.

En torno a 1692 se traslada a Madrid donde será nombrado secretario de la embajada de la Orden Malta en Madrid y seguirá pintando hasta su muerte en 1695.

\section{La obra}

Representa una escena de calle en la que un grupo de niños juegan a los dados sentados en el suelo. A la izquierda un niño acompañado de una muchacha, mira al espectador y señala el grupo de los demás, que parecen estar detenidos en su juego para discutir una cuestión [figura 1].En cuanto a la fecha de realización, la firma a que nos hemos referido establece una frontera temporal; en ningún caso pudo ser pintada antes de $1689^{6}$.

Por otra parte, como han señalado algunos autores, en el Museo de Bellas Artes de Sevilla se conserva un cuadro (El vendedor de vino) en el que aparece un personaje idéntico a uno de los niños de la obra del Prado (Martínez Ripoll, 1982). Se trata del que señala la escena desde la esquina inferior izquierda, con un pedazo de pan en la mano. En el caso de la obra de Sevilla aparece sentado y ofrece una moneda como pago al aguador, pero se trata claramente del mismo modelo. El vendedor de agua se encuentra fechado aunque no es posible distinguir con claridad la totalidad de los dígitos; como hipótesis más plausible se ha estimado que podría corresponder a 1694. Esta fecha lo situaría cerca de 1692, momento en que Villavicencio se traslada a Madrid.

Las dimensiones actuales de Niños jugando a los dados no se corresponden con las originales si no que, muy tempranamente, fue ampliado en su parte superior. La historia de esta manipulación es conocida: en el Inventario Real de 1700 aparece colgado en el Palacio de la Zarzuela formando pareja con otra obra de temática similar, Riña de muchachos ${ }^{7}$, pintada por Luca Giordano [figura 2]. La franja superior que amplía la pintura de Villavicencio frecuentemente se ha atribuido al propio Giordano, que de este modo completa el original y pinta la pareja con la intención decorativa de 
colgarlos juntos ${ }^{8}$. El momento de la intervención no puede ser precisada con exactitud y podría haber tenido lugar incluso en vida del autor (muere en 1695 y Giordano llega a la corte en 1692).

Las medidas de la obra de Villavicencio en la actualidad son 242 x 208 mientras que el original sin el añadido alcanza los $146 \mathrm{~cm}$ de altura. La ubicación de la costura es perfectamente perceptible con luz visible y también es posible diferenciar dos estilos pictóricos diferentes a ambos lados de la misma, pero como se define con mayor claridad y precisión es mediante el estudio de su radiografía.

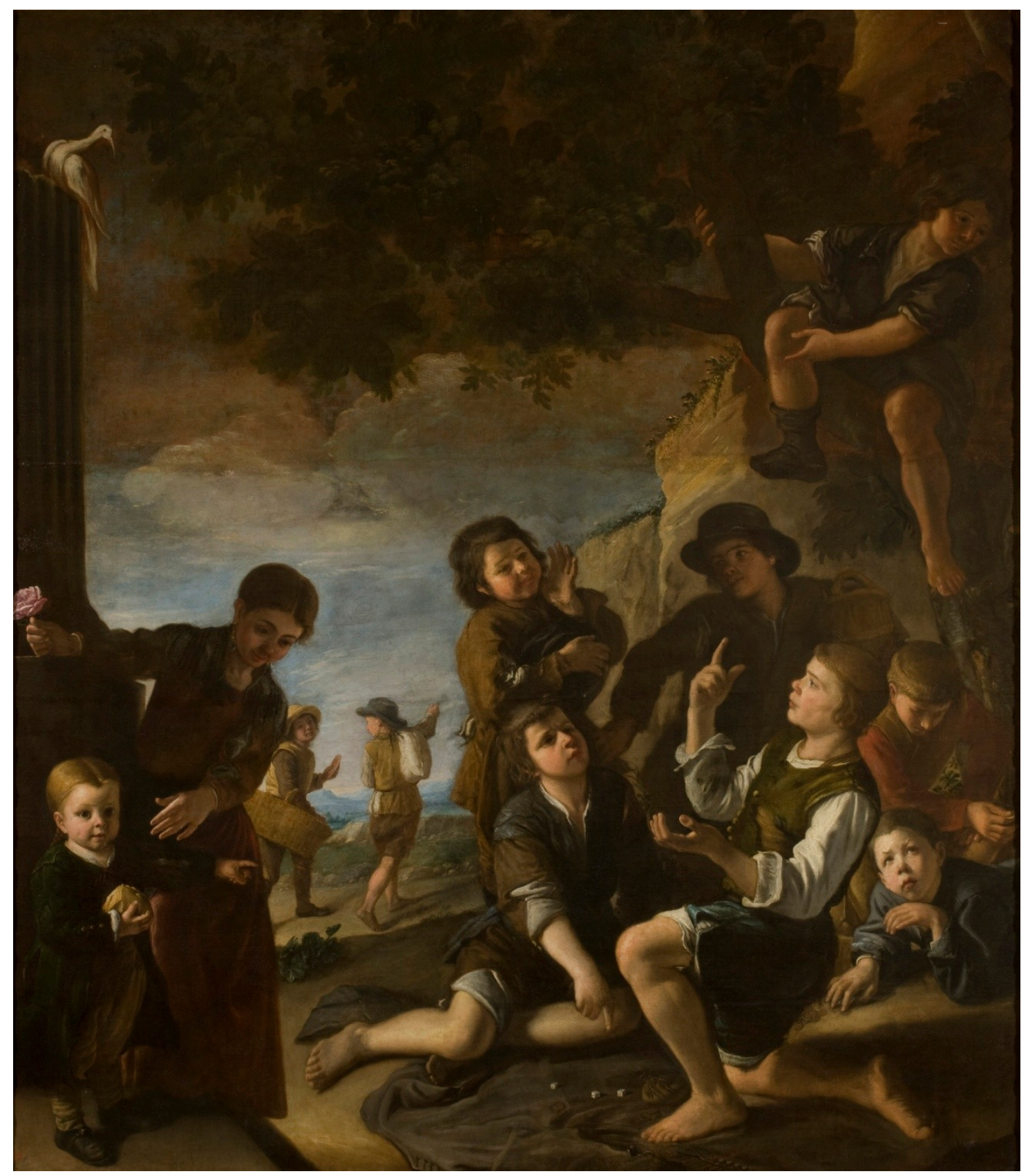

Figura 1. Niños jugando a los dados obra de Pedro Núñez de Villavicencio, ampliada en la zona superior por Luca Giordano (P-01235).

En una colección privada del Reino Unido existe una pintura idéntica a Niños jugando a los dados, pero de menores dimensiones $(45,7$ x 60,4 cm) considerada un "boceto" de la obra del Prado. La escena corresponde a la parte inferior de ésta, sin el añadido de Giordano, y se ajusta a ella en lo 
fundamental, lo que indica que el formato original de Villavicencio era aproximadamente igual al que observamos en la actualidad; únicamente parecen faltar unos pocos centímetros por su parte superior y borde derecho.

Giordano concibe su cuadro como pareja del anterior y esto es patente también en la composición, que se acomoda a ella, tanto en la temática como en su diseño. En ambos casos encontramos una pandilla de niños callejeros jugando, o más bien disputando tras un episodio del juego de dados en un caso o de cartas en el otro. En el caso de la obra de Giordano, más bulliciosa, aparecen varios individuos adultos y se ubica en una calle de la ciudad, mientras que la de Villavicencio transcurre en un escenario más campestre, pero hay varios paralelismos evidentes. Un buen ejemplo de ello son los niños, acompañados de un adulto detrás, que miran al espectador desde la esquina inferior izquierda y señalan la escena, o los de la derecha que tratan de hacerse con el dinero de otro a sus espaldas. Incluso el personaje que se asoma a una ventana de la parte superior, recuerda la forma de la paloma apoyada en la columna donde aparece la firma de Núñez de Villavicencio.

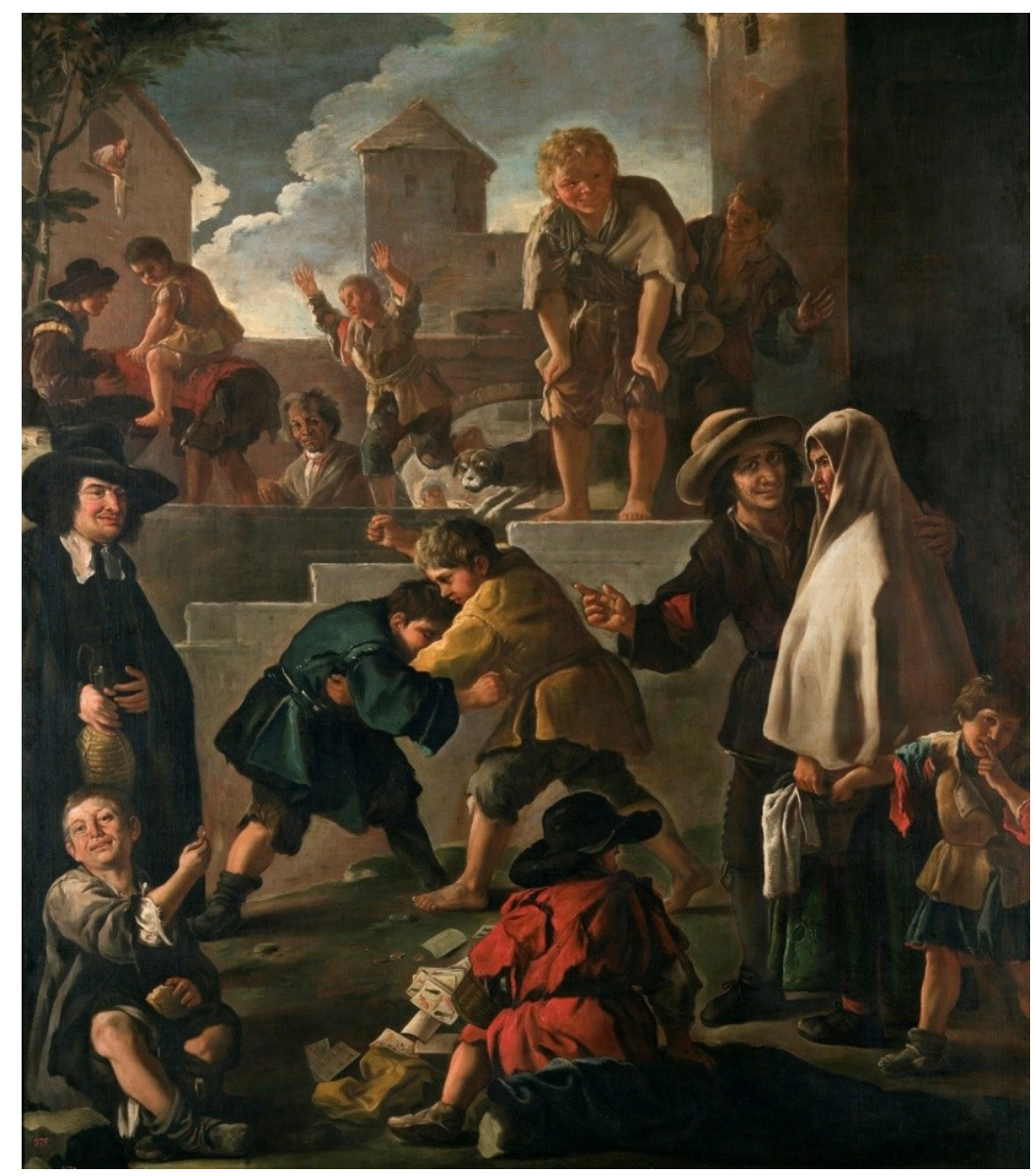

Figura 2. Riña de muchachos de Luca Giordano (P-03939)

Es de sobra conocida la facilidad de Luca Giordano para imitar el estilo de otros artistas (Úbeda, 2008:141-175). En este caso, la imitación le lleva un paso más allá y no sólo se adapta al estilo de Núñez de Villavicencio sino que rinde un homenaje a su maestro, Murillo o incluso al propio Velázquez, evidente en los personajes de la esquina inferior izquierda. De todas formas, los diferentes estilos de ambos artistas quedan en evidencia con una observación detallada de la forma de pintar de cada uno de ellos así como en la radiografía que se comentará más adelante [figura 3]. 


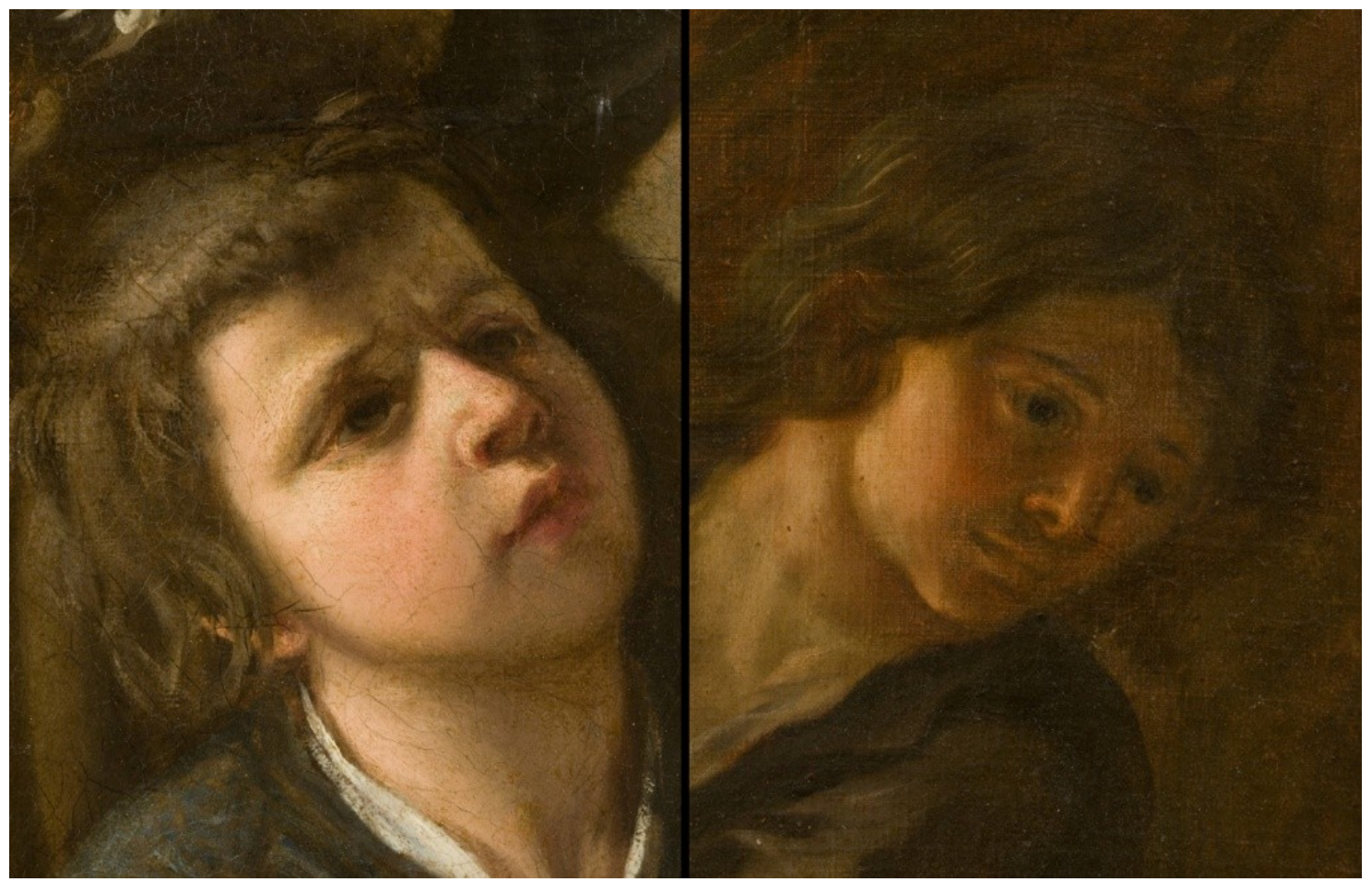

Figura 3. Niños jugando a los dados. A la derecha, detalle del rostro de uno de los niños pintado por Villavicencio y a la izquierda otro de mano de Luca Giordano. Se aprecia con facilidad las diferencias técnicas entre ambos artistas.

La Historia del Arte y la historia material de las obras muestran como, frecuentemente, el tamaño y los formatos de los cuadros ya pintados eran modificados, y habitualmente los encargados de estas transformaciones eran otros pintores. Por lo general las razones que llevaban a ello solían ser estéticas, funcionales o destinadas a reparar una obra en mal estado, y la actitud hacia el original estaba alejada de los criterios de respeto contemplados en la actualidad. De esta manera, era habitual que el pintor introdujera figuras y elementos nuevos en el cuadro terminado, con objeto de conseguir continuidad y disimular la zona añadida. En este caso, Luca Giordano continúa el árbol situado a la derecha y añade el niño que se encuentra subido a él, cuya figura sirve de nexo de unión entre los dos lienzos. Esta misma función tiene la columna colocada en el lado izquierdo.

\section{La radiografía}

El estudio radiográfico de la obra de Núñez de Villavicencio ha aportado gran cantidad de información, no sólo sobre la técnica pictórica de este artista, sino también sino sobre la historia material del cuadro. Además, ha sido posible obtener la radiografía de su pareja Riña de muchachos, lo que ha permitido establecer comparaciones estilísticas y técnicas entre ellas?.

Al examinar el documento radiográfico de Niños jugando a los dados, se aprecia con nitidez el límite del añadido superior de unos $96 \mathrm{~cm}$ de ancho [figura 4], así como las puntadas de la costura que une las dos telas ${ }^{10}$, realizada con hilo grueso y punto sencillo y regular (punto por cima), y el ligamento de tafetán, aunque de diferente densidad, de ambos paños ${ }^{11}$ [figura 5]. La radiografía no permite conocer las medidas exactas del paño de Núñez de Villavicencio, ya que no se aprecian marcas de los travesaños del bastidor, ni huellas en la preparación que indiquen los límites de la escena, ni siquiera líneas en los bordes de la tela. A pesar de ello, como ya se ha explicado en relación con la 
Niños jugando a los dados de Pedro Núñez de Villavicencio. Historia de una obra a través de su radiografía

versión del cuadro recientemente vendido en subasta, es razonable pensar que fuera de un tamaño similar al que presenta en la actualidad.

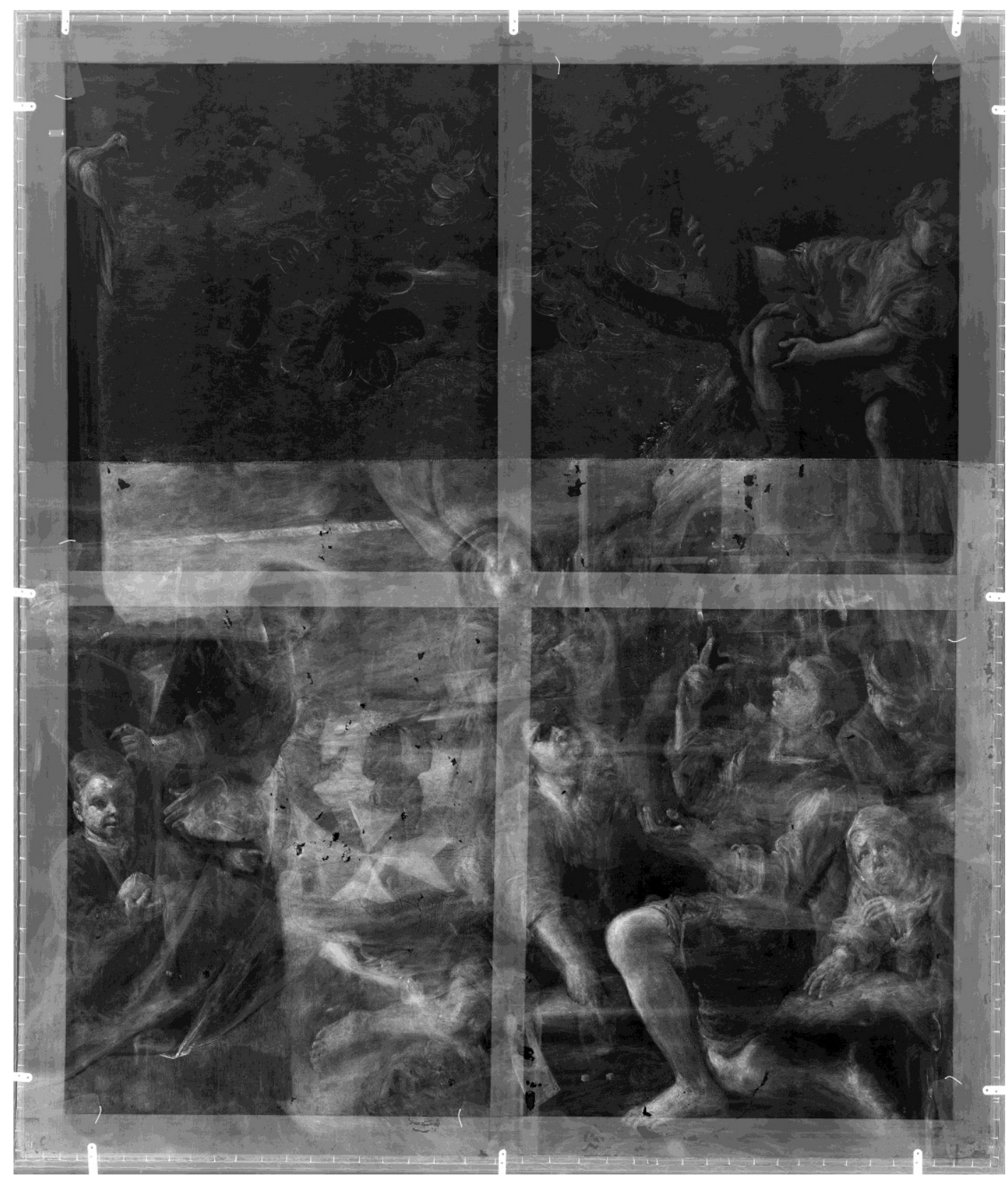

Figura 4. Niños jugando a los dados. La radiografía general muestra la diferente densidad radiográfica entre los lienzos de Villavicencio y Giordano.

El hecho de que Núñez de Villavicencio y Giordano emplearan de manera diferente los materiales, tanto en la capa de preparación como en la pictórica, permite distinguir el añadido con facilidad gracias a la distinta densidad radiográfica. Aunque la capa de preparación ha sido aplicada con brocha en ambos casos, el craquelado que se observa en los paños es muy diferente. En la tela superior se aprecia un craquelado de edad estrecho y pequeño, característico del paso del tiempo en una preparación fina. Por el contrario, el paño central presenta un craquelado prematuro, marcado y ancho, y, puntualmente, craquelados concéntricos o de tela de araña; este tipo de craquelados suelen originarse durante el proceso creativo, ya que en la mayoría de las ocasiones son debidos a la 
superposición de capas sin que haya transcurrido el tiempo de secado necesario entre ellas [figura 6].
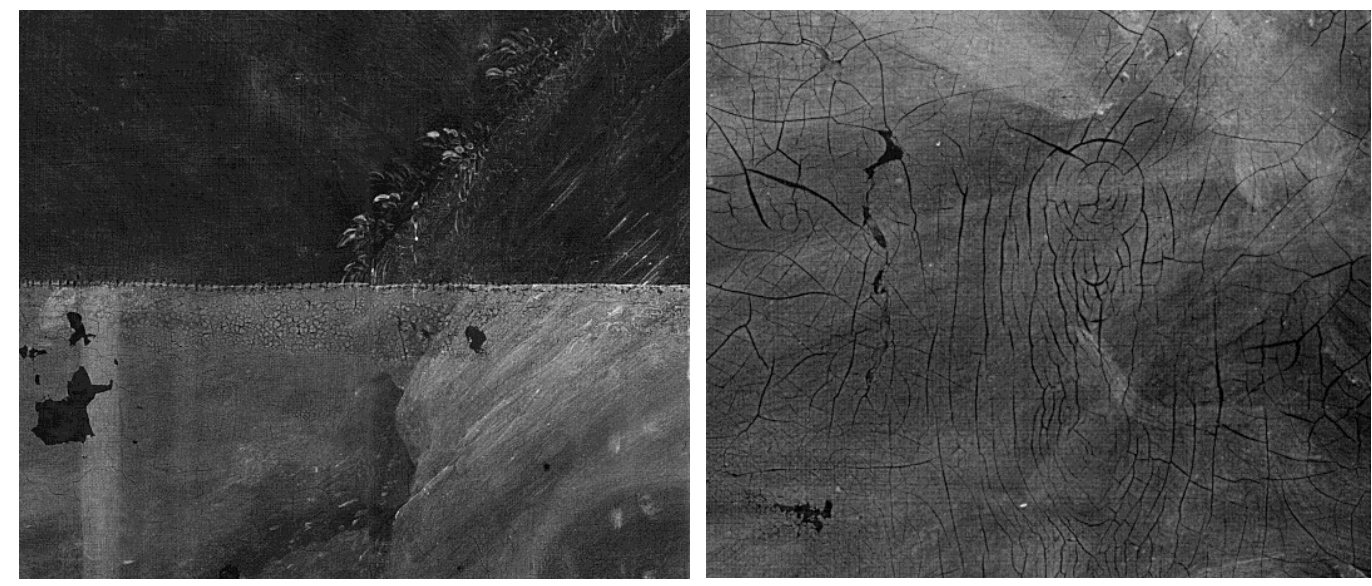

Figuras 5 y 6. Niños jugando a los dados. Detalles en radiografía de la costura que une los paños de los dos artistas y de los craquelados prematuros.

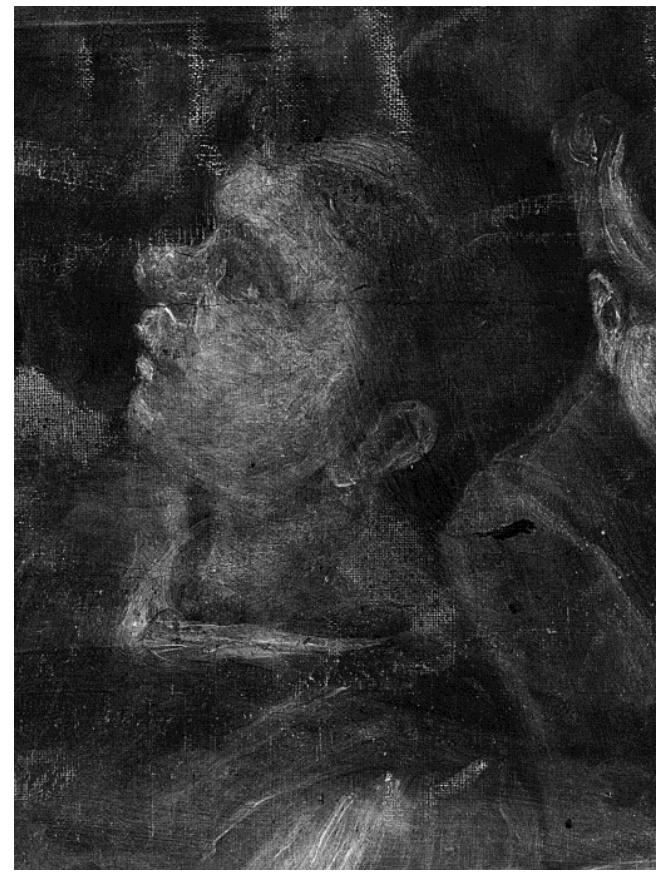

Figura 7. Niños jugando a los dados. Detalle en radiografía de la zona reservada para encajar la cabeza del muchacho.

Al mismo tiempo, queda patente que existen grandes diferencias entre las técnicas pictóricas empleadas por ambos artistas. Núñez de Villavicencio usa pinceladas cuidadas y contenidas, con las que insiste y repasa las formas hasta lograr el efecto buscado. En la radiografía se puede apreciar el empleo de recursos como la reserva de los espacios en los que va a situar las figuras, lo que indica que este pintor cuando comienza a manchar el lienzo, trabaja con una idea previa de la escena en la que cada personaje ocupa un lugar determinado [figura 7]. Por el contrario, tanto en este paño añadido como en la obra a la que se empareja, Riña de muchachos, Giordano trabaja con rapidez, empleando pinceladas amplias y gruesas, sin realizar apenas cambios de composición. Usa el asta del pincel para delimitar contornos, en unas ocasiones en las últimas capas de pintura, como recurso estético, y en otras, en fases previas para encajar elementos de la escena. 
La radiografía del cuadro pintado por Núñez de Villavicencio, permite además apreciar numerosos cambios en la mayoría de las figuras, principalmente para adecuar su tamaño y posición. De esta manera, se ha modificado notablemente la postura de la muchacha que se encuentra a la izquierda de la escena, más erguida en un primer momento. A pesar de la baja densidad radiográfica de las zonas próximas, se pueden observar con claridad modificaciones en la cabeza y en su brazo izquierdo, en un principio más alto y colocado sobre el niño. Es posible que el artista realizara este cambio para acentuar la relación entre estos dos personajes [figura 8].

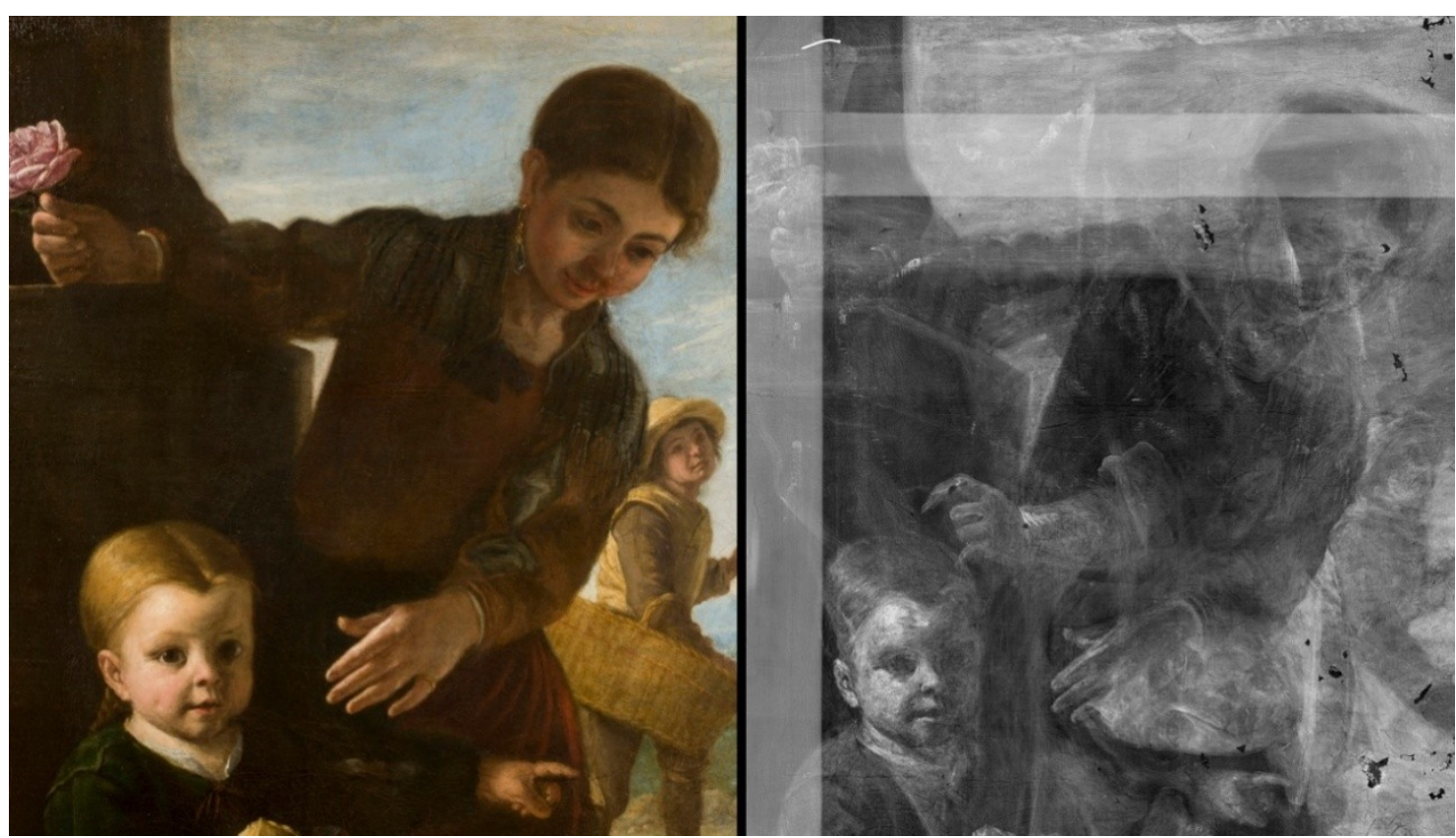

Figura 8. Niños jugando a los dados. Detalle de luz visible y radiografía que muestra los cambios en la posición de la figura de la muchacha, muy evidente en su brazo izquierdo.

Más evidente es la modificación realizada en el muchacho situado en el centro de la escena que ha desplazado hacia la izquierda y colocado en una posición más elevada, proporcionando un mayor espacio al juego de los dados [figura 9]. Se observa con claridad el primer esbozo de la cabeza, del tronco y del brazo y pierna derecha, cuya ejecución se abandonó en una fase temprana sin determinar detalles y calidades.

También se aprecian cambios en el niño situado en la esquina inferior derecha, tanto en su identidad (en un principio parecía haberse pintado una niña con la cabeza cubierta); como en la posición de sus manos y el diseño de los pliegues de la ropa [figura 10]. De todos los cambios realizados por Núñez de Villavicencio en esta figura, quizás el más interesante sea el del brazo izquierdo que modifica varias veces: en un primer momento aparecía estirado con la mano apoyada sobre el suelo, próxima a las monedas, más tarde dobla el codo del personaje y apoya su mejilla sobre la palma de esta mano; para finalmente pintar la palma de la mano boca abajo de manera que la barbilla reposa sobre ella. El niño que situado de pie sujeta un sombrero entre sus brazos, parecía llevar otro objeto, posiblemente un saco y tener el brazo derecho más bajo. En el resto de las figuras se observan pequeñas modificaciones para ajustar la posición y proporciones de algunos elementos, como en el brazo izquierdo, la pierna derecha o el perfil del rostro del muchacho arrodillado en primer término. 


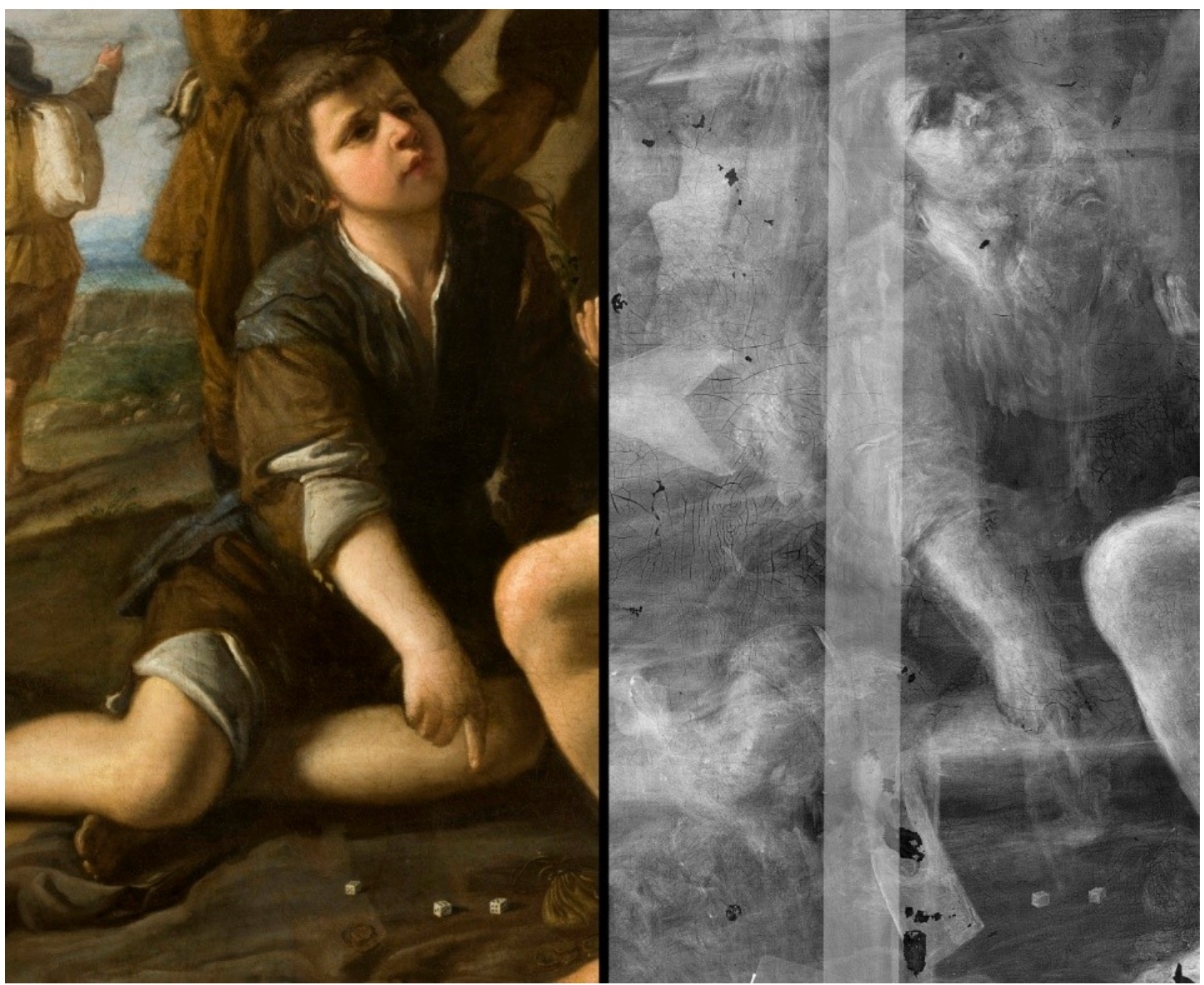

Figura 9. Niños jugando a los dados, detalle de luz visible y radiografía. Se observa cómo Pedro Núñez de Villavicencio ha modificado la ubicación de esta figura, desplazándola hacia arriba.

En definitiva, la mayoría de las modificaciones realizadas por Núñez de Villavicencio tienen la intención de dar naturalismo al movimiento de los personajes, a sus posiciones y a las relaciones que se establecen entre ellos. Únicamente el desplazamiento del muchacho sentado en el centro de la escena, parece obedecer al deseo de otorgar un mayor protagonismo al bodegón formado por la bolsa de dinero, las monedas y los dados que dan nombre a este cuadro. Estos cambios indican que el artista fue transformando la escena a medida que avanzaba el cuadro, plasmando sus ideas iniciales, sus dudas y las soluciones que adoptaba para mejorar la composición ${ }^{12}$.

Una vez analizada esta escena, una observación más detallada de la radiografía de este paño, permite apreciar pinceladas que no se corresponden con la obra definitiva. Al girar la radiografía $90^{\circ}$ a la derecha, se descubre que Pedro Núñez de Villavicencio ha reutilizado un lienzo en el que había pintado un retrato de cuerpo entero de un caballero cuyo rostro, con el cabello largo, puede observarse con claridad [figura 11]. Esta figura se encuentra de pie y en posición frontal aunque ligeramente girada hacia la izquierda, y tanto en el pecho como en la capa que cae de su hombro izquierdo se han pintado sendas cruces de Malta. Mientras la mano derecha del personaje se apoya en una mesa y sostiene lo que parece ser un pliego de papel doblado, la mano izquierda sujeta un par de guantes. En el fondo, detrás de la figura se observan los pliegues de una gran cortina. 


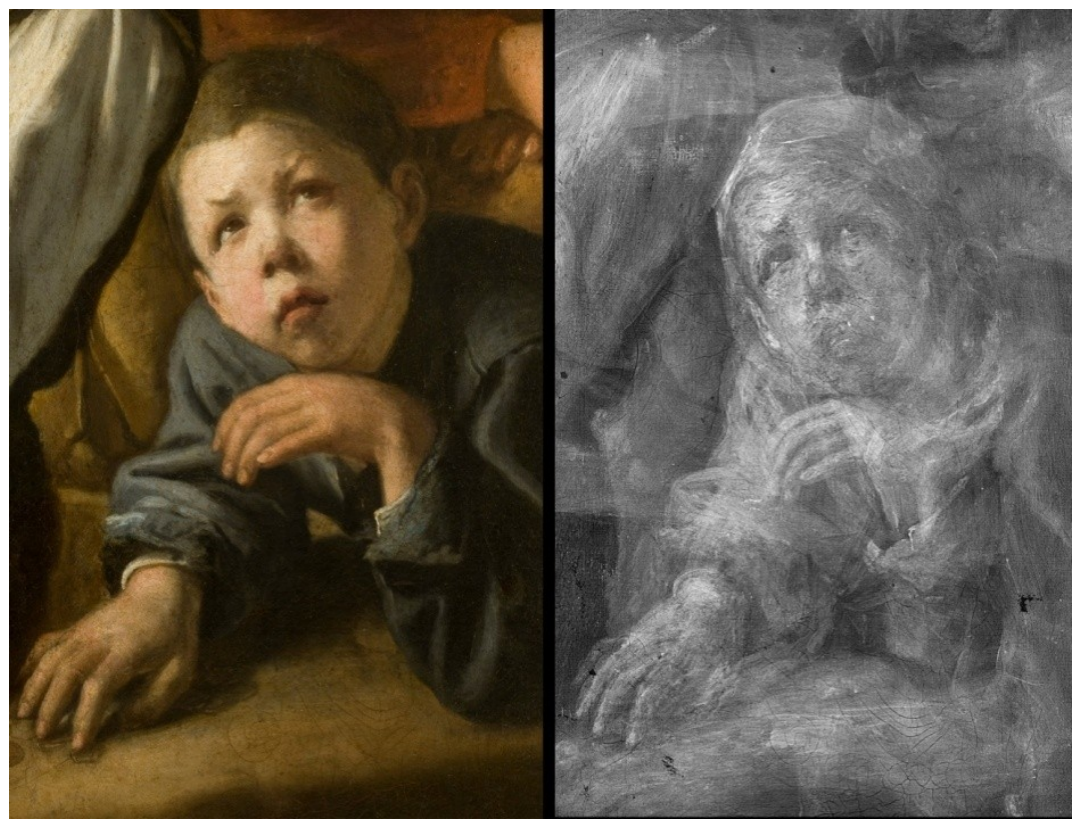

Figura 10. Niños jugando a los dados. Detalle de luz visible y radiografía que permite apreciar los numerosos cambios que se han realizado en esta figura.

Esta reutilización del lienzo y la existencia de varias capas de pintura superpuestas justifica el ancho craquelado prematuro que se observa y que permite deducir que no debió transcurrir mucho tiempo desde que se pinta y abandona el retrato del caballero y se decide reutilizarlo para la escena de los niños, pudiendo datar la primera composición en una fecha cercana a 1689-90.

Por otro lado, la reutilización de una tela ya preparada y pintada explicaría el hecho de que no se observen en los bordes actuales del lienzo, las deformaciones del tensado inicial ni las marcas de la preparación sobre el bastidor. De esta manera las dimensiones de la escena en la que aparece este retrato no parecen ser las originales, ya que, además, en el lateral derecho se observa una silla con tachuelas colocada de perfil, además de otros elementos incompletos. Por alguna razón, no debió convencerle lo pintado o bien el encargo se disolvió porque el artista tomó el lienzo, lo recortó, lo giró $90^{\circ}$ y lo reutilizó.

\section{Primera hipótesis: el personaje}

El personaje que aparece bajo la escena de Niños jugando a los dados debió ser una persona de alto rango, como lo demuestra el hecho de ser retratado de cuerpo entero, apoyado en un bufete y con los guantes en la mano izquierda. En la derecha sujeta un papel, relacionado en los retratos protocolarios con las tareas de responsabilidad en la administración. Su edad podría estar en torno a los 40-50 años: no es un anciano ni tampoco un joven, es un caballero de edad madura, corpulento y que luce una abundante cabellera. Su porte es altivo y majestuoso, consciente de su rango. Se trata por tanto, de un eminente caballero de la orden de Malta, con cargo administrativo y que debe estar posando para Núñez de Villavicencio unos años antes (no muchos, como ya hemos explicado) de pintar Niños jugando a los dados, es decir en la segunda mitad de los años 80. 

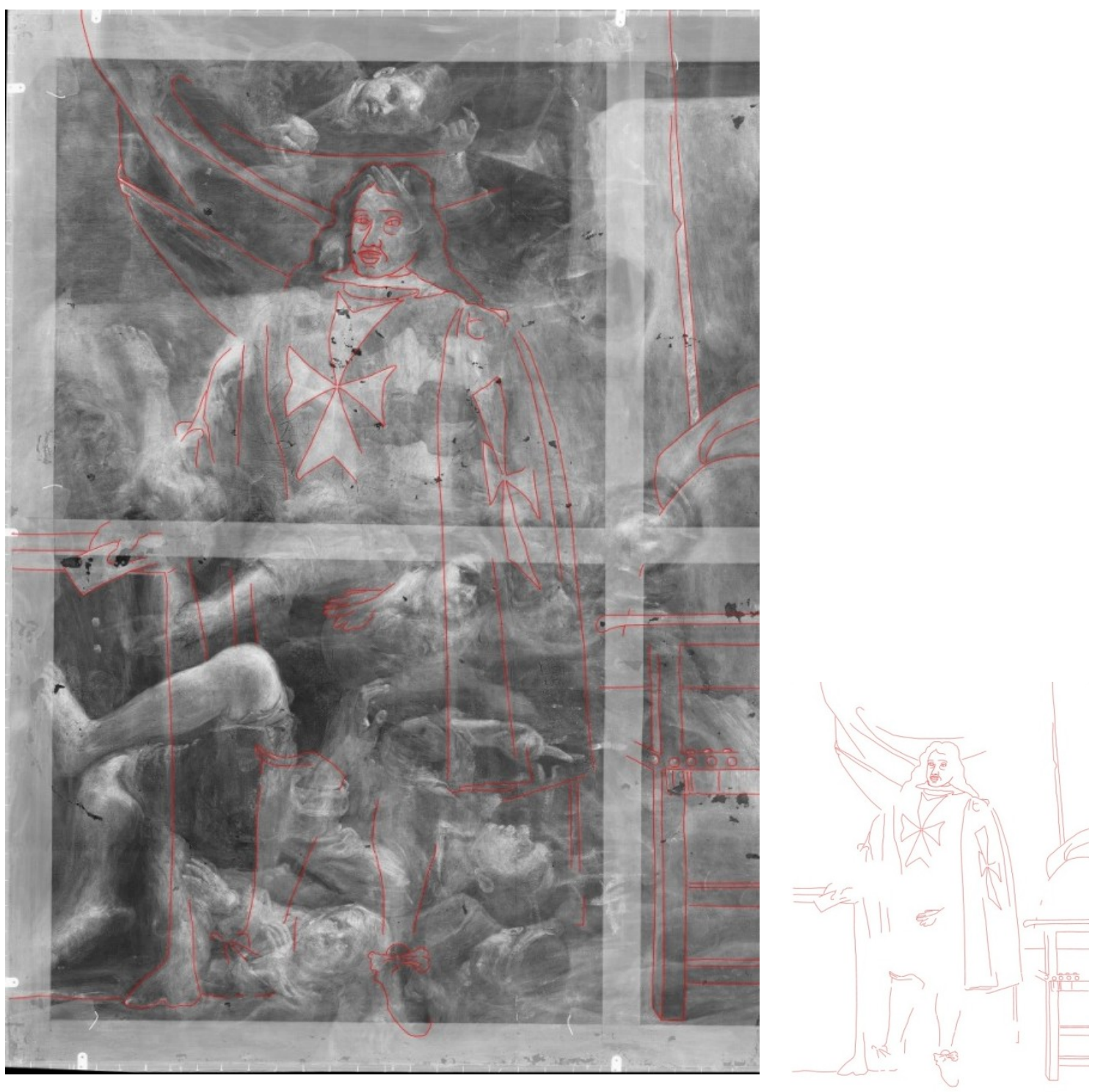

Figura 11. Niños jugando a los dados. Silueteado de la figura del caballero que se observa en la radiografía y que actualmente se encuentra oculta por la escena de los niños.

Volviendo a la biografía de Núñez de Villavicencio, a lo largo de su vida pudo tener contacto con numerosos caballeros de características similares a las mencionadas. Siendo él mismo un destacado miembro de la Orden de Malta, su relación con los personajes ilustres de la misma pudo ser muy directa. Sin embargo hay un nombre con el que se relacionó a lo largo de toda su carrera y, que especialmente en sus inicios como pintor, tuvo una gran importancia: Don Manuel Arias Porres.

Manuel Arias fue un caballero destacado de la Orden, en la que ocupó distintos cargos. En el momento de llegar Villavicencio a Malta, era Vicecanciller de la misma, además de titular de varias encomiendas. Con cargo a una de ellas, la Orden concede una pensión vitalicia a Núñez de Villavicencio lo que le supondrá una fuente de ingresos constante. Arias está además muy relacionado con la actividad artística en la isla, participando en la creación de una academia donde coincidirán Mattia Pretti y Villavicencio. Su afición a las artes se plasma en el hecho de que, a su vuelta a España en 1684, traiga consigo numerosas pinturas, varias de ellas de mano del italiano ${ }^{13}$.

La relación de Manuel Arias con Núñez de Villavicencio se prolongará toda su vida y probablemente llegaron a ser amigos personales. Siempre favoreció al pintor y le ayudó en sus aspiraciones políticas, sin descuidar las suyas propias, acumulando numerosos e importantes cargos 
a lo largo de su vida ${ }^{14}$. En 1690 se había ordenado sacerdote, a la edad de 55 años y en 1702 será nombrado arzobispo de Sevilla, donde acometió importantes proyectos desde el punto de vista artístico (Quiles y Cano, 2006: 48-49). Muerto Carlos II también jugará un papel importante en el proceso de la sucesión.

La importancia del personaje es por tanto indudable y también su relación con Núñez de Villavicencio, una relación que mantuvo durante toda su vida y probablemente llegó a ser personal. Su edad y las fechas de su biografía cuadran perfectamente con el personaje representado; a finales de los años 80 reside en España y ostenta un cargo de importancia.

En el palacio arzobispal de Sevilla se guarda una colección de retratos de todos los arzobispos de la diócesis a lo largo de los siglos incluido uno de D. Manuel Arias Porres (Falcón, 1997: 364-387). Este retrato pertenece por tanto a su época como arzobispo, es decir, debió ser realizado a partir de 1702, cuando contaba con una edad aproximada de 66 años. Si contemplamos esta imagen junto a la del caballero pintado por Villavicencio [figura 12], el parecido no es inmediato pero sí razonable: el arzobispo es un hombre mayor, más delgado, con el pelo corto y sin bigote, pero al igual que el caballero resulta ligeramente cargado de hombros y corpulento. Una observación atenta revela que la forma de los ojos, el arco de las cejas, la nariz, el mentón y sobre todo la boca carnosa son muy similares en ambos $\operatorname{casos}^{15}$. Sin abandonar el terreno de las hipótesis, las biografías de Arias y el pintor y el parecido entre ambas caras nos permite plantear que se trate del mismo sujeto.

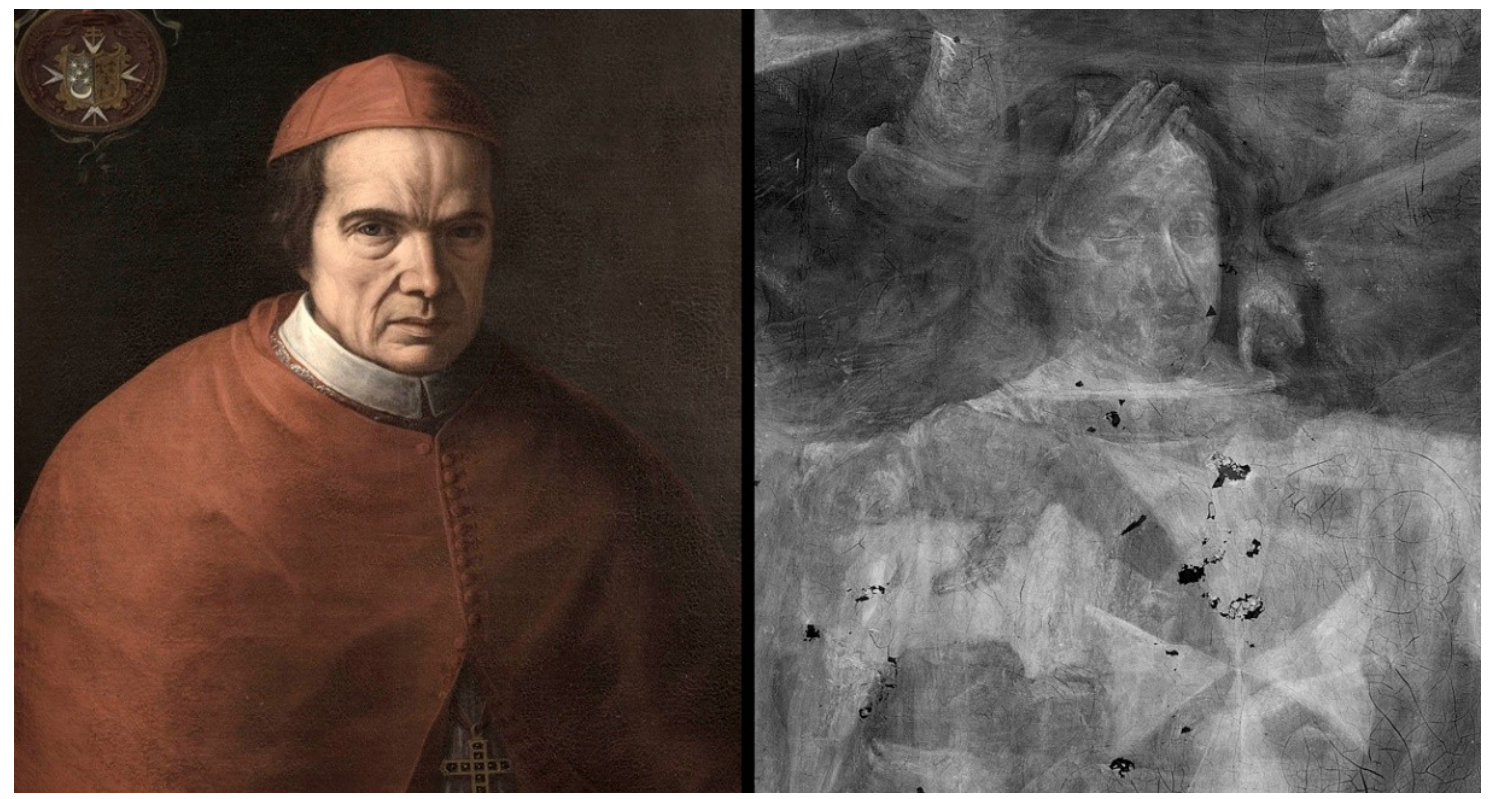

Figura 12. Comparación entre el rostro del caballero que aparece en radiografía de Niños jugando a los dados y el retrato de Don Manuel Arias Porres como arzobispo de Sevilla, obra perteneciente a la colección de El Palacio Arzobispal de Sevilla. La radiografía del rostro del caballero ha sido rotada en sentido horizontal para facilitar la comparación. Catedral de Sevilla. Biblioteca Capitular. Fotógrafo: Luís Serrano.

En cuanto a las razones por las que el retrato pudo ser desechado, podemos encontrar dos, de distinta índole. En 1690, D. Manuel Arias se ordena sacerdote e inicia una rápida carrera dentro de la jerarquía eclesiástica. Quizá, si el retrato estaba en proceso de acabado por esa fecha, ya no le pareció conveniente esa imagen de aparato con la indumentaria de la Orden. También es posible que el resultado final no resultara satisfactorio desde el punto de vista compositivo o estético, ya que, en cierto modo, la figura del caballero resulta poco armoniosa, quizá de hombros demasiado anchos para el tamaño de la cabeza (ver figura 11). 


\section{Segunda hipótesis: ¿un retrato doble?}

Volviendo a la radiografía del retrato de cuerpo entero, es fácil apreciar que la escena continuaba hacia la derecha. Además de la silla, se distinguen los pliegues de una tela que, por la altura y el tamaño, podrían ser parte de la vestimenta de una segunda figura. Es posible por tanto que Villavicencio hubiera pintado un cuadro de mayores dimensiones en el cual aparecerían dos figuras retratadas, a la izquierda el caballero con la cruz de Malta y a la derecha otro personaje del cual sólo se observa parte del atuendo.

Generalmente, en los retratos dobles existe una relación entre los retratados, pero en este caso el caballero mira hacia la izquierda, ajeno a la presencia de la otra figura. Además, los pliegues de la vestimenta del segundo personaje le sitúan demasiado lejos; no existe por tanto contacto entre ellos ni físico ni visual. La composición del espacio y el estudio de la forma y ubicación de los pliegues, así como la perspectiva y tamaño de la línea blanca continua en posición oblicua que encontramos cerca del límite recortado, permiten apuntar una hipótesis sobre la escena que pintó en origen Villavicencio: la figura representada a la derecha podría ser un pintor, que sobre un gran lienzo (la línea blanca que hemos citado correspondería al canto del bastidor), está retratando al caballero que posa para él con gran solemnidad.

El hecho de que aparezca la silla cerca del pintor, en la que podría incluso estar sentado, indicaría su posición social. Avanzando un poco más en la hipótesis, ¿por qué no pensar que el pintor fuera el propio Villavicencio, "caballero pintor", retratando a su amigo? La relación entre ambos, como ya hemos expuesto, fue estrecha y D. Manuel Arias siempre apoyó, incluso económicamente, su actividad artística. En el año 1684 el caballero regresa de Malta en calidad de embajador de la Orden en Madrid y muy bien pudo encargar un retrato en su nuevo destino, de mano de su compañero de Orden y amigo, Núñez de Villavicencio, que quiso rendirle un homenaje pintándose junto a él.

En definitiva, la escena de Niños jugando a los dados oculta por tanto una compleja historia material que ha sido posible reconstruir a través del estudio de su radiografía. Esto pone de manifiesto la importancia de este tipo de técnicas que dan acceso a información de gran valor más allá de lo visible.

\section{Agradecimientos}

Las autoras quieren agradecer su ayuda al personal del Museo Nacional del Prado que ha colaborado en la elaboración de este trabajo, especialmente a Jaime García-Máiquez, Inma Echeverría, Elena Arias, Javier Portús y Alberto Otero.

\section{Notas}

[1] Especialmente completa y bien documentada es la biografía del pintor escrita por González Ramos, en la que nos vamos a basar principalmente.

[2] Por ejemplo, la otra pintura de mano de Núñez de Villavicencio que conserva el Museo Nacional del Prado, La Piedad con la Magdalena (P-6934), es posiblemente una copia de un original perdido del maestro italiano.

[3] La pintura original se conserva en una colección particular madrileña, una réplica del mismo artista en el Museo Calvet de Avignon y un grabado de 1681, de mano de Collin, en el museo de Amberes. También existen dos copias en el Museo de Bellas Artes de Sevilla y en el palacio Arzobispal de Sevilla.

[4] González Ramos transcribe el documento de la Real Orden de Malta en el apéndice documental de su libro, p. 234 
[5] La firma, en el pedestal de la columna de la izquierda dice lo siguiente: $\operatorname{Fr} D P^{o}$ de villavicencio fabn // Comor. de vodonal hispalis

[6] Algunos autores han citado el Inventario Real del Alcázar de Madrid de 1686, localizando la obra en el obrador de los pintores de cámara. Sin embargo, consultado dicho inventario, no hemos encontrado referencia alguna a su presencia en esta estancia.

[7] Propiedad del Museo del Prado, depositada en la Real Academia de Jurisprudencia y Legislación (P-03939).

[8] Aunque algunos autores han disentido con respecto a esta cuestión, en general parece aceptada y, en nuestra opinión, como se verá después, se trata de un hecho cierto.

[?] Estas radiografías se han realizado en el Gabinete de Documentación Técnica del Área de Restauración del Museo Nacional del Prado. Condiciones de trabajo: P-01235; 55kv, 7mA, 1,8 min, $570 \mathrm{~cm}$. P-03939; 50kv, $7 \mathrm{~mA}, 1,3 \mathrm{~min}, 480 \mathrm{~cm}$. Equipo de la marca Seifert modelo Eresco 160 MFR2.

[10] La costura presenta aproximadamente 35 puntadas cada $10 \mathrm{~cm}$.

[11] Mientras que el paño de Villavicencio tiene unos 12 hilos verticales y 12 horizontales $/ 1 \mathrm{~cm} 2$, el de Giordano presenta 16 verticales y 14 horizontales, siendo por tanto más densa y de trama más cerrada.

[12] Los numerosos cambios que se observan en la radiografía de Niños jugando a los dados, nos hacen pensar que el "boceto" de la colección particular británica que reproduce fielmente la escena final de Villavicencio, podría ser en realidad una copia de la obra una vez terminada, que se habría realizado antes de que Luca Giordano añadiera la banda superior.

[13] Estas obras son actualmente, propiedad del Palacio Arzobispal, la catedral y el Museo de Bellas Artes de Sevilla. En este viaje vino también la obra de Villavicencio Judith mostrando la cabera de Holofernes al pueblo de Betulia, probablemente regalada por el artista a Manuel Arias y conservada en el Museo de Bellas Artes de Sevilla.

[14] Ejerció de embajador de la Real Orden de Malta en Madrid, de Gran Prior de la Orden en Castilla y posteriormente, en 1693 es nombrado presidente del Consejo de Castilla

[15] Hay que tener en cuenta también que lo que estamos viendo es una radiografía, no una imagen visible, y que la posición y expresión del retratado varía ligeramente de uno a otro

\section{Bibliografía}

FALCÓN MARQUEZ, T. (1997). El Palacio Arzobispal de Sevilla. Sevilla: CajaSur.

GONZALEZ RAMOS, R. (1999). Núñę de Villavicencio, caballero pintor. Sevilla: Diputación de Sevilla.

MARTínEZ RIPOLL, A. (1982). "Para una cronología de Pedro Núñez de Villavicencio", Goya, 169-171: 105-112.

PALOMINO, A. (1715-1724). El Museo Pictórico y Escala Óptica. V. III. El Parnaso Español Pintoresco y Laureado. (Ed. 1988, Madrid: Aguilar).

ÚBEDA DE LOS COBOS, A. (2008). Luca Giordano y el Casón del Buen Retiro. Madrid: Museo Nacional del Prado.

QUILES GARCIA, F. y CANO RIVERO, I. (2006). Bernardo Lorente Germán y la pintura sevillana de su tiempo (1680-1759). Madrid: Fernando Villaverde Ediciones. 
Inventarios Reales en 12 Volúmenes y un Índice (Transcripción fotocopiada). Biblioteca del Museo Nacional del Prado.

\section{Laura Alba}

Museo Nacional del Prado

Paseo del Prado, s/n. 28014 Madrid España

laura.alba@museodelprado.es

Laura Alba trabaja desde 2003 en el Gabinete de Documentación Técnica del Museo Nacional del Prado en el ámbito de los estudios técnicos, especializada principalmente en el análisis radiográfico de las obras de arte. Licenciada en Bellas Artes (Especialidad Restauración de Pintura) por la Universidad Complutense de Madrid, ha trabajado para diferentes fundaciones y museos como la Fundación Juan March, la Fundación ICO, el Museo de Arte Abstracto Español, el Museo Provincial de Cuenca y el Museo Guggenheim de Bilbao.

\section{Maite Jover de Celis}

Museo Nacional del Prado

Paseo del Prado, s/n. 28014 Madrid España

maite.jover@museodelprado.es

Maite Jover de Celis trabaja en el Laboratorio de Análisis del Área de Restauración del Museo Nacional del Prado. Licenciada en Ciencias Biológicas y Diplomada en Conservación y Restauración de Bienes Culturales (Especialidad Pintura) por la Escuela de Madrid, ha trabajado en varias instituciones relacionadas con la conservación como el Centro de Conservación y Restauración de Bienes Culturales de Castilla y León o el Instituto del Patrimonio Cultural de España, siempre dentro del ámbito de los estudios técnicos de obras de arte. 
